\title{
Localized cytokine responses to total knee arthroplasty and total knee revision complications
}

Nicole Prince ${ }^{1,2}$, Julia A. Penatzer ${ }^{1,2}$, Matthew J. Dietz ${ }^{2}$ and Jonathan W. Boyd ${ }^{2,3^{*}}$

\begin{abstract}
Background: The study of localized immune-related factors has proven beneficial for a variety of conditions, and one area of interest in the field of orthopaedics is the impact of implants and localized infections on immune response. Several cytokines have shown increased systemic concentrations (in serum/plasma) in response to implants and infection, but tissue-level cytokines have not been investigated as thoroughly.

Methods: This exploratory study investigated tissue-level cytokines in a cohort of patients $(N=17)$ in response to total knee arthroplasty and total knee revision to better understand the immune response to implants and localized infection (e.g., prosthetic joint infection). The overall goal of this study was to provide insight into the localized cytokine response of tissues and identify tissue-level markers specific to inflammation caused by implants vs. inflammation caused by infection. Tissues were collected across several anatomical locations and assayed with a panel of 20 human inflammatory cytokines to understand spatial differences in cytokine levels.
\end{abstract}

Results: In this study, six cytokines were elevated in implanted joints, as compared to native joints: IL-10, IL-12p70, IL-13, IL-17A, IL-4, and TNF- $a(p<0.05)$. Seven cytokines showed infection-dependent increases in localized tissues: IL-1 $a, I L-1 \beta, I L-6, I L-8, M C P-1, M I P-1 a$, and MIP-1 $\beta$ ( $p<0.05)$.

Conclusions: This study demonstrated that differences exist in tissue-level cytokines in response to presence of implant, and some cytokines were specifically elevated for infection; these responses may be informative of overall tissue health. These results highlight the utility of investigating localized cytokine concentrations to offer novel insights for total knee arthroplasty and total knee revision procedures, as well as their complications. Ultimately, this information could provide additional, quantitative measurements of tissue to aid clinical decision making and patient treatment options.

Keywords: Interleukin, Infection, Inflammation, Total knee arthroplasty, Total knee revision, Spatial distribution

\section{Background}

The inflammatory response of tissues involves a series of biological events regulated by a number of immune factors, and the actions of these immune factors are partially reliant on the cytokines and chemokines produced

${ }^{*}$ Correspondence: Jonathan.boyd@hsc.wvu.edu

2 Department of Orthopaedics, West Virginia University School of Medicine, Morgantown, WV, USA

Full list of author information is available at the end of the article in response to pathogens, foreign bodies, and other stimuli [1-3]. These responses are of interest to the field of orthopaedics, especially with regard to the immune response to implants, infection, and chronic inflammation [4-6]. An elevated immune response has been observed following total knee arthroplasty (TKA) procedures; increased levels of cytokines, particularly interleukin (IL)-1, IL-4, IL-6, IL-10, and tumor necrosis factor alpha (TNF- $\alpha$ ), have been shown both on a systemic level (i.e., serum/plasma) as well as on a more localized level 
(i.e., synovial fluid) [7-9]. However, many aspects of this response are not well understood. For example, a majority of TKA procedures are successful, but implant-related and infection-related complications can negatively affect a patient's quality of life. Properly addressing these issues is of high priority to the field of orthopaedics, especially considering the increasing demand for joint replacement [10]. Many studies have noted the pain, inflammation, and dissatisfaction that can occur following these procedures, affecting approximately $20 \%$ of patients undergoing TKA $[11,12]$, but it is not entirely known what role cytokines play in this chronic inflammatory response. Infections, such as prosthetic joint infection (PJI), are another serious complication and are a leading cause of total joint failure [13]. PJI is a localized infection surrounding a prosthetic joint and can result following implantation, often necessitating surgical intervention [14]. PJI is a major concern following TKA/total knee revision (TKR) procedures and can be difficult to treat. The infections are often persistent and unable to be resolved using conventional methods, presenting a challenge for clinicians [15]. The systemic immune response to PJI has been studied extensively, but the localized tissue response is not as well understood. In order to better understand the immune response to implants and localized infection, this study investigated levels of 20 inflammatory cytokines in localized tissue surrounding the joint. While defining the localized response to implants and infection can be difficult [7-9], localized cytokine responses have been investigated for other pathological conditions. A few studies have characterized localized cytokine responses in trauma [16-18] and respiratory infection [19], and these studies demonstrated that the local cytokine environment differs when compared to systemically circulating levels. Currie et al. [16] showed that differences in cytokine concentrations exist in skeletal muscle samples in a spatially-dependent manner using an animal model of traumatic injury. Similarly, Hauser et al. [18] observed differences in levels of cytokines at the site of injury compared to systemic levels in response to trauma in humans. Other research groups have observed spatially-related differences of other immune-related factors for stroke [20], and in response to allergens [21] in animal models. These studies introduced the concept of using immune markers on a localized level to better understand these conditions.

TKA and TKR procedures trigger inflammatory cascades, initiating cytokine responses and elevating systemic cytokine concentrations; higher levels of cytokines have been observed following these surgeries. The elevation in cytokine levels has been attributed to the trauma of surgery as well as the introduction of implants into the body [22, 23]. However, this inflammation is sometimes prolonged, which can cause major complications for patients. The causes of chronic inflammation following these procedures are still unknown, and resolution of the inflammation is challenging [24]. Therefore, understanding the changes in inflammatory response specific to implant-related inflammation is beneficial to improving the outcome of these individuals.

Similarly, the localized response to PJI has not been characterized to understand the local immune modulation in these cases. Many studies have investigated systemically circulating levels of interleukins and other cytokines for their roles in infection, and several cytokines are used as diagnostics of PJI [25-27]. Several studies have specifically focused on the utility of measuring IL-6 and IL-8 levels in serum for diagnosing and monitoring PJI, both of which have increased specificity over conventional methods; this knowledge has greatly benefitted the clinical treatment options for PJI [28, 29]. However, PJI remains one of the most serious complications following revision knee arthroplasty. In fact, infection is one of the most common causes for revision, being implicated in $20.4 \%$ of all revision TKA procedures between 2009 and 2013 [30]. While defining the systemic response to sepsis and infection has paved the way for improved diagnostics [31-33], less is known about the environment of localized infections and what role cytokines play in determining tissue health.

The present study focused on understanding differences in localized distributions of cytokines in TKA and TKR procedures, with and without presence of infection, using PJI as the model for localized infections. The ultimate goal of this study was to characterize the immune modulation on a tissue level that occurs in response to joint implantation and infection to better understand localized tissue health. The information gained could aid clinical management of these complications by narrowing down cytokines that are indicative of response to PJI. It represents the first known investigation of tissue-level cytokines in response to implant-related and infectionrelated complications, to our knowledge.

\section{Patients, materials, and methods}

\section{Patients}

Following Institutional Review Board (IRB) approval (IRB Protocol \#1709745853) and patient consent, six patients undergoing primary total knee arthroplasty (TKA) and 11 patients undergoing total knee revision (TKR) procedures participated in the study ( 8 males, 9 females; aged 45-82 years; body max index [BMI] 24.6-43.7). Subjects were recruited over a 12-month period. All six primary TKA patients were undergoing elective surgery for total replacement of the knee joint with a diagnosis of osteoarthritis. At the time of this study, this was the first 
arthroplasty procedure on either knee joint. In the TKR group, patients were further characterized into aseptic and septic revision procedures. Patients with aseptic revisions $(\mathrm{N}=5)$ were undergoing revisions due to failures of the prosthetic joint but did not show presence of infection. For ease of the reader, samples from these patients will be referred to as aseptic TKR tissues. Patients with septic revisions $(\mathrm{N}=6)$ met clinical criteria for a PJI diagnosis as defined by the Musculoskeletal Infection Society (MSIS) criteria [13]. Samples from these patients will be referred to as septic TKR tissues. All six patients diagnosed with PJI were tissue culture positive: four tested culture positive for Staphylococcus epidermidis, one for Methicillin-sensitive Staphylococcus aureus (MSSA), and one for Enterobacter cloacae. More patient information can be found in Table 1. Systemic C-reactive protein (CRP) levels in serum are additionally listed as reference.

\section{Collection of tissue samples}

All TKA and TKR procedures were performed by a single surgeon with standard debridement and washing protocols. Tissues were collected at a total of four distinct anatomical locations, broadly characterized into two tissue layers: four adjacent tissue layer (ATL) samples and three radial tissue layer (RTL) samples. The ATL samples came from the initial debridement. Tissues from the ATL layer were closer to the knee joint (or prosthetic implant). Conversely, RTL samples were taken from a tissue layer further removed from the joint (or prosthetic implant) after the surgeon completed debridement. The difference in depth of the RTL tissues and ATL tissues was approximately $5-10 \mathrm{~mm}$ and was dependent on the individual patient. Measurements were made from point of origin to standardize tissue samples taken between patients. Tissues were taken at four anatomical locations illustrated in Fig. 1. Briefly, the solid line circle represents location (1) medial femoral condyle (F); the dashed line circle represents location (2) medial tibial plateau (T); the solid line square represents location (3) lateral gutter (LG); and the dashed line square represents location (4) posterior capsule (PC). Anatomical locations 1-4 were collected for the ATL layer, and locations 1-3 were collected for the RTL layer. Location 4, PC, could not be taken in the RTL layer due to proximity to neurovascular structures. Therefore, a total of seven tissue samples were taken for each patient.

\section{Sample preparation}

Tissues were collected during TKA and TKR procedures in the operating room and immediately stored on dry ice. Once all tissues had been collected for an individual patient, they were washed with $1 \times$ cold phosphate-buffered saline (PBS) to remove blood and debris. Tissues were grossly dissected using a scalpel to remove scar tissue or cement, then stored at $-80^{\circ} \mathrm{C}$. When samples had been collected for all patients, tissues were thawed on ice

Table 1 Patient information

\begin{tabular}{|c|c|c|c|c|c|c|}
\hline ID & Sex & TKA/TKR & BMI $\left(\mathrm{kg} / \mathrm{m}^{2}\right)$ & Diabetic (Y/N) & CRP (mg/L) & Culture \\
\hline P1 & $\mathrm{F}$ & TKA & 33.8 & $\mathrm{~N}$ & N/A & Negative \\
\hline P2 & $\mathrm{F}$ & TKA & 39.8 & $\mathrm{~N}$ & N/A & Negative \\
\hline P3 & $F$ & TKA & 39.8 & $\mathrm{~N}$ & N/A & Negative \\
\hline P4 & M & TKA & 29.7 & Y & N/A & Negative \\
\hline P5 & M & TKA & 24.6 & $N$ & N/A & Negative \\
\hline P6 & M & TKA & 27.2 & $N$ & N/A & Negative \\
\hline F1 & $\mathrm{F}$ & TKR-aseptic & 28.2 & $N$ & 4.3 & Negative \\
\hline F2 & F & TKR-aseptic & 29.8 & $N$ & 0.2 & Negative \\
\hline F3 & F & TKR-aseptic & 33.9 & N & $<1$ & Negative \\
\hline F4 & M & TKR-aseptic & 40.4 & Y & 3.6 & Negative \\
\hline F5 & M & TKR-aseptic & 26.2 & N & 2.1 & Negative \\
\hline F6 & $\mathrm{F}$ & TKR-septic & 43.7 & N & 28.8 & S. epidermidis \\
\hline F7 & $\mathrm{F}$ & TKR-septic & 30.8 & Y & 161.4 & S. epidermidis \\
\hline F8 & $\mathrm{F}$ & TKR-septic & 41.9 & $N$ & 21.7 & E. cloacae \\
\hline F9 & M & TKR-septic & 36.2 & $N$ & 33.5 & MSSA \\
\hline F10 & M & TKR-septic & 33.8 & Y & 3.8 & S. epidermidis \\
\hline F11 & $M$ & TKR-septic & 31.9 & $\mathrm{~N}$ & 111.9 & S. epidermidis \\
\hline
\end{tabular}

Six primary TKA and 11 revision TKR patients were enrolled in the study, creating a heterogenous cohort of males and females varying in age (45-82 years) and comorbidities. Primary TKA patients have ID format P\#; revision TKR patients have ID format F\#. This table lists general patient information including the pathogen for which each septic patient tested culture-positive following testing on the day of surgery. Serum CRP values were obtained pre-operatively in the revision setting. Cultures were obtained from intraoperative tissue samples 


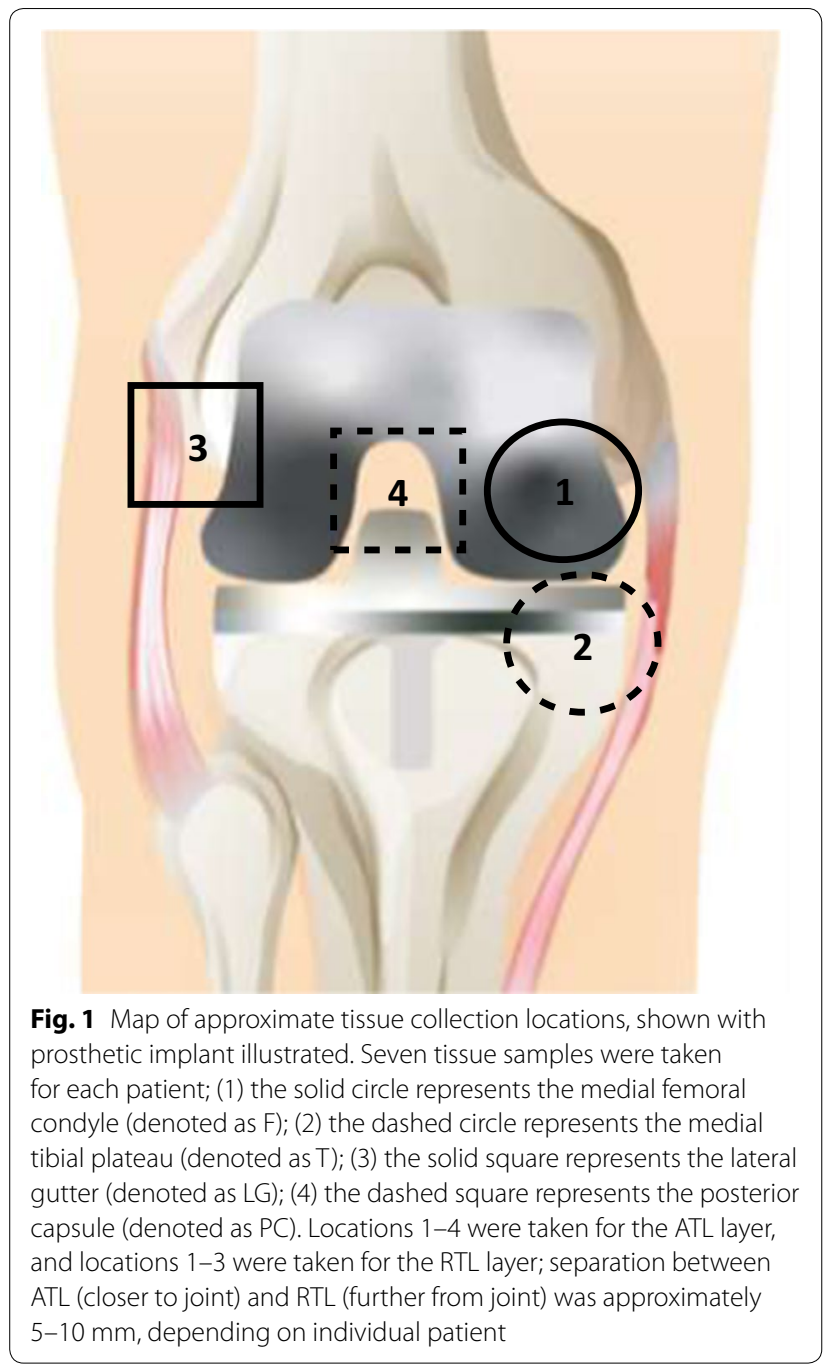

and cut into sections approximately $30 \mathrm{mg}$ in size; tissues were homogenized by sonication in $500 \mu \mathrm{L}$ cell lysis solution (Bio-Rad, Hercules, CA) containing $20 \mathrm{mM}$ phenylmethylsulfonyl fluoride (Sigma-Aldrich, St. Louis, MO). Protein extraction was performed using methods adapted from Hulse et al. [34]. Thawed samples were vortexed for $1-3 \mathrm{~s}$ and centrifuged at $5000 \times g$ for $5 \mathrm{~min}$ at $4{ }^{\circ} \mathrm{C}$. The supernatant was collected and tested for total protein content using a Pierce BCA Protein Assay Kit (Thermo Scientific, Waltham, MA), according to manufacturer's instructions. Absorbance values for total protein content were determined on an Infinite M1000 multimode plate reader (Tecan, Raleigh, NC).

\section{Cytokine measurement}

To standardize samples for total protein content, tissue homogenates were individually diluted to a total protein concentration of $900 \mu \mathrm{g} / \mathrm{mL}$ with cell lysis buffer (BioRad). Cytokine quantification was performed using a magnetic bead-based multiplex Inflammation Human ProcartaPlex panel assay (Invitrogen, Carlsbad, CA) and measured using a Bio-Plex 200 suspension array system and Pro II Wash Station (Bio-Rad), according to the manufacturer's instructions.

\section{Statistical analysis}

Data were analyzed using Prism 5 (GraphPad, San Diego, CA) and SAS JMP (Cary, NC). Standard curves were generated for each protein using either a four- (4PL) or fiveparameter logistic (5PL) regression model, depending on the individual protein. Cytokine concentrations were determined using standard curve interpolation, then corrected by dilution factor to compare tissue homogenates. Cytokine concentrations are expressed as picograms of cytokine per milliliter of tissue homogenate $(\mathrm{pg} / \mathrm{mL})$. Samples with fluorescence intensity values below the lower limit of quantitation (LLOQ) or above the upper limit of quantitation (ULOQ) were omitted from statistical comparisons. Outliers were identified using the $1.5 \times$ interquartile range (IQR) rule and omitted from analysis. Two-way analysis of variance (ANOVA) with Bonferroni's post-test was used to determine significant differences between primary TKA, aseptic TKR, and septic TKR tissue samples at each tissue location. Each tissue homogenate was tested in duplicate for cytokine concentration. Data are expressed as the mean \pm standard error of the mean (SEM).

Quadratic discriminant analysis was conducted to evaluate the combined capacity of cytokine response to predict the state of tissue. Using SAS JMP, all measured responses were cast as covariates, and the "group" was assigned as a classification category (primary TKA, aseptic TKR, septic TKR). The Shrink Covariances option was applied to account for the different covariances within the categories. Quadratic discriminant analysis is a predictive modeling tool, and when there are a large number of variables compared to observations, as is the case in this study, Shrink Covariances is frequently employed to improve the stability and reduce prediction variance [35]. This analysis included 13 covariates; only those cytokines that produced statistically significant two-way ANOVA comparisons for either infection-specific or implantspecific comparisons were included: IL- $1 \alpha$, IL-1 $\beta$, IL-6, IL-8, monocyte chemoattractant protein (MCP)-1, macrophage inflammatory protein (MIP)-1 $\alpha$, MIP-1 $\beta$, IL-10, IL-12p70, IL-13, IL-17A, and TNF- $\alpha$. Biplot rays are plotted to indicate how each covariate influences the canonical space, with the direction and magnitude signifying the degree of association with the respective group (primary TKA, aseptic TKR, septic TKR).

Due to the limited sample size, this study was not able to control for age, sex, BMI, or other comorbidities. 
(See figure on next page.)

Fig. 2 Seven cytokines showed infection-dependent elevation in localized tissues. Individual tissue locations are shown for all groups. Two-way ANOVAs with Bonferroni's post-test were conducted to test for significant differences between groups at each individual location $(p<0.05)$. Significant differences between groups at a particular location are marked as: ${ }^{*}$ denotes significant difference from primary TKA $(N=6)$; \# denotes significant difference from aseptic TKR $(N=5)$; \% denotes significant difference from septic TKR $(N=6)$; all symbols denote significance at the $p<0.05$ level

Pearson correlations were run between cytokine concentrations and age, sex, and BMI for each patient to analyze the contribution of these variables. Bonferroni's correction was applied to correct for multiple inferences, as previously described by Bland et al. [36].

\section{Results}

Changes in cytokine concentrations were observed for comparisons of primary TKA vs. aseptic TKR vs. septic TKR tissues. Overall, cytokine concentrations were generally elevated in TKR (both septic and aseptic) compared to TKA, and septic TKR exhibited higher cytokine levels than aseptic TKR for several cytokines. Seven cytokines (IL-1 $\alpha$, IL-1 $\beta$, IL-6, IL-8, MCP-1, MIP-1 $\alpha$, and MIP-1 $\beta$ ) showed increased concentrations in septic TKR tissues compared to both aseptic TKR tissues and primary TKA tissues $(\mathrm{p}<0.05)$. Six cytokines (IL-10, IL-12p70, IL-13, IL-17A, IL-4, and TNF- $\alpha$ ) showed differences in concentration between primary TKA and TKR (both aseptic and septic) $(\mathrm{p}<0.05)$, but these six cytokines were not significantly different between aseptic TKR and septic TKR. These comparisons are described in detail over the following sections. Additional human inflammatory cytokines were tested, but they did not produce statistically significant comparisons in this study: E-Selectin, granulocyte-macrophage colony-stimulating factor (GM-CSF), interferon-alpha (IFN- $\alpha$ ), interferon-gamma (IFN- $\gamma)$, and interferon gamma-induced protein 10 (IP-10).

\section{Seven cytokines exhibited infection-specific elevation in localized tissues}

Seven cytokines showed an increase in concentration that was dependent on the presence of localized infection: IL-1 $\alpha$, IL-1 $\beta$, IL-6, IL-8, MCP-1, MIP-1 $\alpha$, and MIP-1 $\beta(\mathrm{p}<0.05)$. For these cytokines, primary TKA averages were lowest, with an increase in aseptic TKR and further increase in septic TKR. For IL- $1 \alpha$, the average concentration of primary TKA tissues was $1.1 \mathrm{pg} / \mathrm{mL}$, and rose to $11.8 \mathrm{pg} / \mathrm{mL}$ in aseptic TKR; the concentration was elevated to $30.3 \mathrm{pg} / \mathrm{mL}$ in septic TKR. Locationspecific differences are marked in Fig. 2, and it is clear that most significant comparisons are present within locations of the ATL layer. IL- $1 \beta$ showed a similar trend, with a mean of $1.7 \mathrm{pg} / \mathrm{mL}$ in primary TKA tissues, which rose to $5.4 \mathrm{pg} / \mathrm{mL}$ in aseptic TKR, and further elevated to
$39.1 \mathrm{pg} / \mathrm{mL}$ in septic TKR. For IL-1 $\beta$, the most marked differences between groups came from comparisons of locations ATL LG and ATL PC (Fig. 2). IL-6 followed, with an average of $8.5 \mathrm{pg} / \mathrm{mL}$ in primary TKA, rising to $24.2 \mathrm{pg} / \mathrm{mL}$ in aseptic TKR, and finally $610.7 \mathrm{pg} / \mathrm{mL}$ in septic TKR. Location ATL PC showed the most dramatic increase in concentration in septic TKR compared to other groups (Fig. 2). IL-8 levels were $7.6 \mathrm{pg} / \mathrm{mL}$ in primary TKA, which increased to $91.1 \mathrm{pg} / \mathrm{mL}$ in aseptic TKR, and rose to $553.9 \mathrm{pg} / \mathrm{mL}$ in septic TKR. Differences in IL-8 were significant between at least two groups at all locations besides ATL LG and ATL PC at $\mathrm{p}<0.05$ (note: ATL PC for IL-8 not shown due to omission of outliers). For MCP-1, the average of primary TKA tissues was $113.0 \mathrm{pg} / \mathrm{mL}$, which increased to $258.8 \mathrm{pg} / \mathrm{mL}$ for aseptic TKR, and further increased to $565.1 \mathrm{pg} / \mathrm{mL}$ for septic TKR. RTL LG showed the most significant comparisons between groups for MCP-1 (Fig. 2, $\mathrm{p}<0.05$ ). MIP- $1 \alpha$ followed the same trend, with an average of $7.8 \mathrm{pg} / \mathrm{mL}$ for primary TKA, which rose to $27.8 \mathrm{pg} / \mathrm{mL}$ in aseptic TKR, and was elevated to $81.6 \mathrm{pg} / \mathrm{mL}$ in septic TKR. ATL locations showed the most significant increases in MIP- $1 \alpha$ between groups (Fig. 2, $\mathrm{p}<0.05$ ). For MIP-1 $\beta$, primary TKA tissues showed an average of $21.3 \mathrm{pg} / \mathrm{mL}$ and were increased to $46.0 \mathrm{pg} / \mathrm{mL}$ for aseptic TKR and further increased to $123.4 \mathrm{pg} / \mathrm{mL}$ in septic TKR. Locations ATL $\mathrm{T}$ and ATL PC showed the most significant increases between groups for MIP-1 $\beta$ (Fig. 2, $\mathrm{p}<0.05$ ). As shown in Fig. 2, cytokine concentrations in the ATL layer locations were generally higher than the RTL layer locations.

\section{Six cytokines exhibited implant-related elevation (primary TKA vs. aseptic/septic TKR)}

Six cytokines, IL-10, IL-12p70, IL-13, IL-17A, IL-4, and TNF- $\alpha$, exhibited higher levels in TKR tissues as compared to primary TKA tissues at a minimum of one location $(\mathrm{p}<0.05)$. In other words, there were significant differences $(\mathrm{p}<0.05)$ between primary TKA and aseptic/septic TKR, but there were no significant differences between aseptic TKR and septic TKR. For IL-10, the average value in primary TKA was $0.9 \mathrm{pg} / \mathrm{mL}, 8.4 \mathrm{pg} /$ $\mathrm{mL}$ in aseptic TKR, and $6.6 \mathrm{pg} / \mathrm{mL}$ in septic TKR. All locations showed significantly different comparisons to aseptic TKR and septic TKR (Fig. 3, p<0.05). With the same general trend, IL-12p70 had an average of $5.7 \mathrm{pg} /$ $\mathrm{mL}$ in primary TKA, $30.7 \mathrm{pg} / \mathrm{mL}$ in aseptic TKR, and 


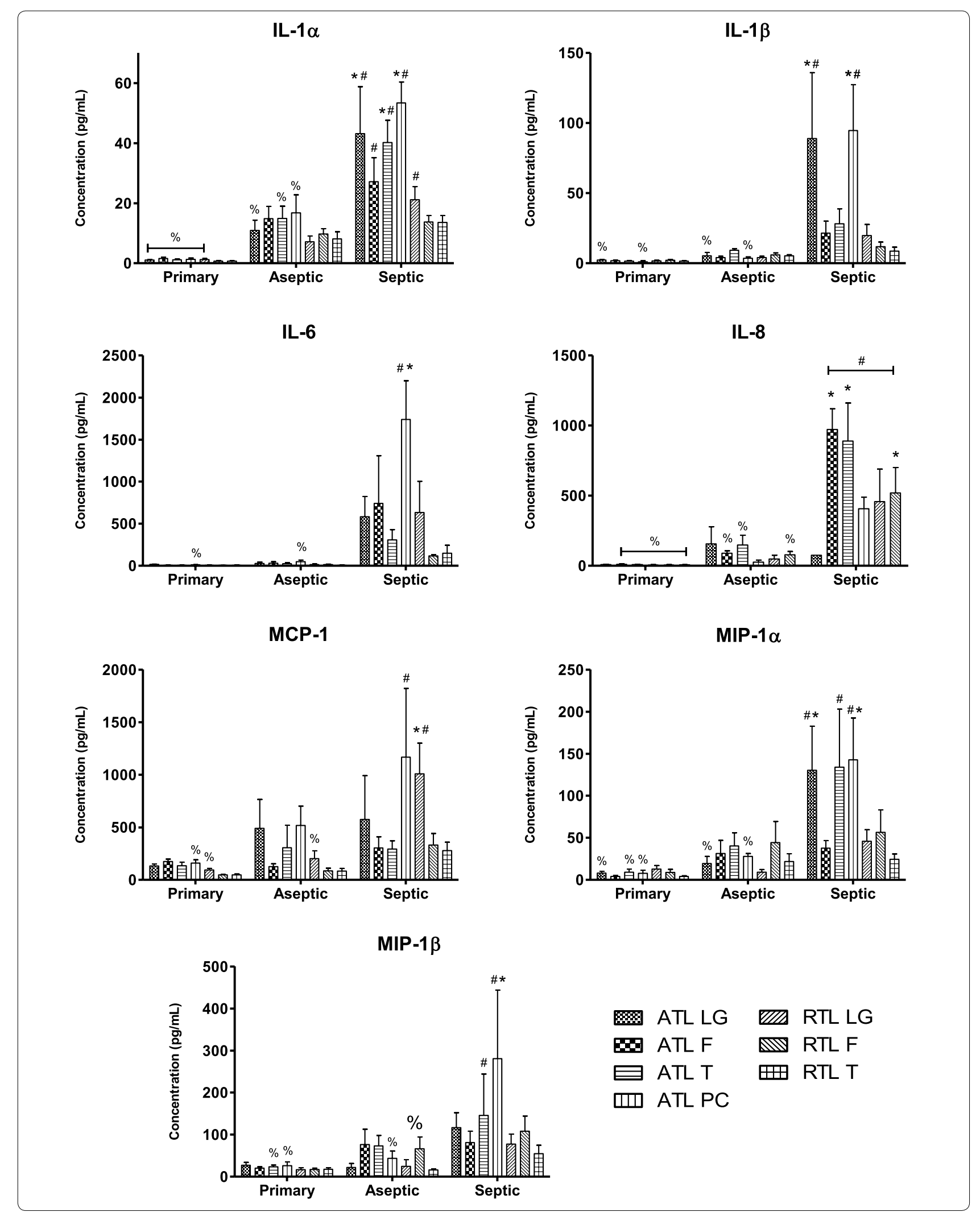




\section{(See figure on next page.)}

Fig. 3 Six cytokines showed implant-related elevation in localized tissues that was not infection-dependent. Individual tissue locations are shown for all groups. Two-way ANOVAs with Bonferroni's post-test were conducted to test for significant differences between groups at each individual location $(p<0.05)$. Significant differences between groups at a particular location are marked as: ${ }^{*}$ denotes significant difference from primary TKA $(N=6)$; \# denotes significant difference from aseptic TKR $(N=5)$; \% denotes significant difference from septic TKR $(N=6)$; all symbols denote significance at the $p<0.05$ level

$20.7 \mathrm{pg} / \mathrm{mL}$ in septic TKR. However, IL-12p70 only showed one statistically significant comparison $(\mathrm{p}<0.05)$ at the ATL PC location between primary TKA and aseptic TKR (Fig. 3). For IL-13, the average in primary TKA was $1.8 \mathrm{pg} / \mathrm{mL}, 9.6 \mathrm{pg} / \mathrm{mL}$ in aseptic TKR, and $9.9 \mathrm{pg} /$ $\mathrm{mL}$ in septic TKR. Locations ATL F, ATL T, and RTL F exhibited significant comparisons between groups (Fig. 3, $\mathrm{p}<0.05)$. Following this trend, IL-17A average concentrations were $5.3 \mathrm{pg} / \mathrm{mL}$ in primary TKA, $16.3 \mathrm{pg} / \mathrm{mL}$ in aseptic TKR, and $18.9 \mathrm{pg} / \mathrm{mL}$ in septic TKR. All locations except RTL T showed significant comparisons (Fig. 3, $\mathrm{p}<0.05$ ). For $\mathrm{IL}-4$, average concentration in primary TKA was $6.9 \mathrm{pg} / \mathrm{mL}$, which rose to $19.6 \mathrm{pg} / \mathrm{mL}$ in aseptic TKR, and further to $24.8 \mathrm{pg} / \mathrm{mL}$ in septic TKR. Again, all locations except RTL T showed significant comparisons (Fig. 3, p<0.05). Finally, TNF- $\alpha$ followed the same trend, with an average concentration of $16.9 \mathrm{pg} / \mathrm{mL}$ in primary TKA, $71.1 \mathrm{pg} / \mathrm{mL}$ in aseptic TKR, and $86.8 \mathrm{pg} / \mathrm{mL}$ in septic TKR. All locations except RTL T showed significant comparisons (Fig. 3, p <0.05).

\section{Quadratic discriminant analysis (QDA) revealed distinct cytokine profiles for TKA vs. TKR}

The two-way ANOVA comparisons of cytokines between different groups revealed seven cytokines that showed infection-specific elevation (beyond inflammation caused by implants), and six cytokines that showed increases due to implants, but not infection (Figs. 2 and 3). To further probe the structure of these cytokine profiles between groups, quadratic discriminant analysis was conducted. These 13 cytokines were included as covariates. The analysis classified the combined observed responses into predetermined groups of primary TKA, aseptic TKR, and septic TKR. The group was predicted based on the covariate responses associated with each group, respectively. For each group, all seven locations were included for all individuals in that group, which means there were 42 counts for primary TKA (7 tissue locations, 6 patients), 35 values for aseptic TKR (7 tissue locations, 5 patients), and 42 counts for septic TKR (7 tissue locations, 6 patients). In total, of 119 counts, only 8 were misclassified, indicating a good prediction ability of the model. All 8 misclassifications were errors of a prediction of aseptic TKR group, when the values were originally from the septic TKR group. In other words, these individuals were falsely classified as aseptic based on cytokine profiles while they were actually septic. Further, there is overlap between the $95 \%$ confidence intervals for cytokine profiles of aseptic TKR and septic TKR patients (Fig. 4), which may be responsible for the misclassification.

\section{Effects of age, sex, and BMI on cytokine concentrations}

The research presented here did not control for age, sex, or BMI due to the limited sample size of this exploratory study. To better understand the connections between cytokines of interest (IL-1 $\alpha$, IL-1 $\beta$, IL-6, IL-8, MCP-1, MIP-1 $\alpha$, MIP-1 $\beta$, IFN- $\gamma$, IL-10, IL-13, IL-17A, IL-4, and TNF- $\alpha$ ) and these factors, Pearson correlations were run and analyzed for statistical significance. When the Bonferroni's correction was applied, as described in [36], none of the correlations between cytokine levels and age, sex, or BMI were significant $(p>0.05)$, but the correlations are displayed in Table 2 for transparency. Although there is an established connection in the literature between inflammatory cytokine levels and age, sex, and BMI, the lack of significant Pearson correlation p-values indicates these were not confounding variables for this study [37-39].

\section{Discussion}

Inflammation in response to implants and infection following TKA/TKR procedures remains a serious complication and is a high priority for clinicians. However, not much is known about the local immune response of the tissue surrounding the implant/infection. While a variety of cytokines (and other biomarkers) have been researched from a systemic view [40, 41], their clinical use is still debated [31-33, 42]. Further, the cytokine responses have not been as well characterized on a localized tissue level. The tissue-level cytokine response may add further understanding of the localized environment and could give insight into tissue health that would aid clinicians in the management of these post-surgical complications through surgical debridement. Tissue-level cytokines have been measured with respect to spatial gradients in traumatic injury [16-18], respiratory infection [19], stroke [20], and allergic response [21], and these studies provided useful information regarding the respective immune responses. These have established a basis for this study to investigate the localized implantrelated and infection-specific tissue responses. 


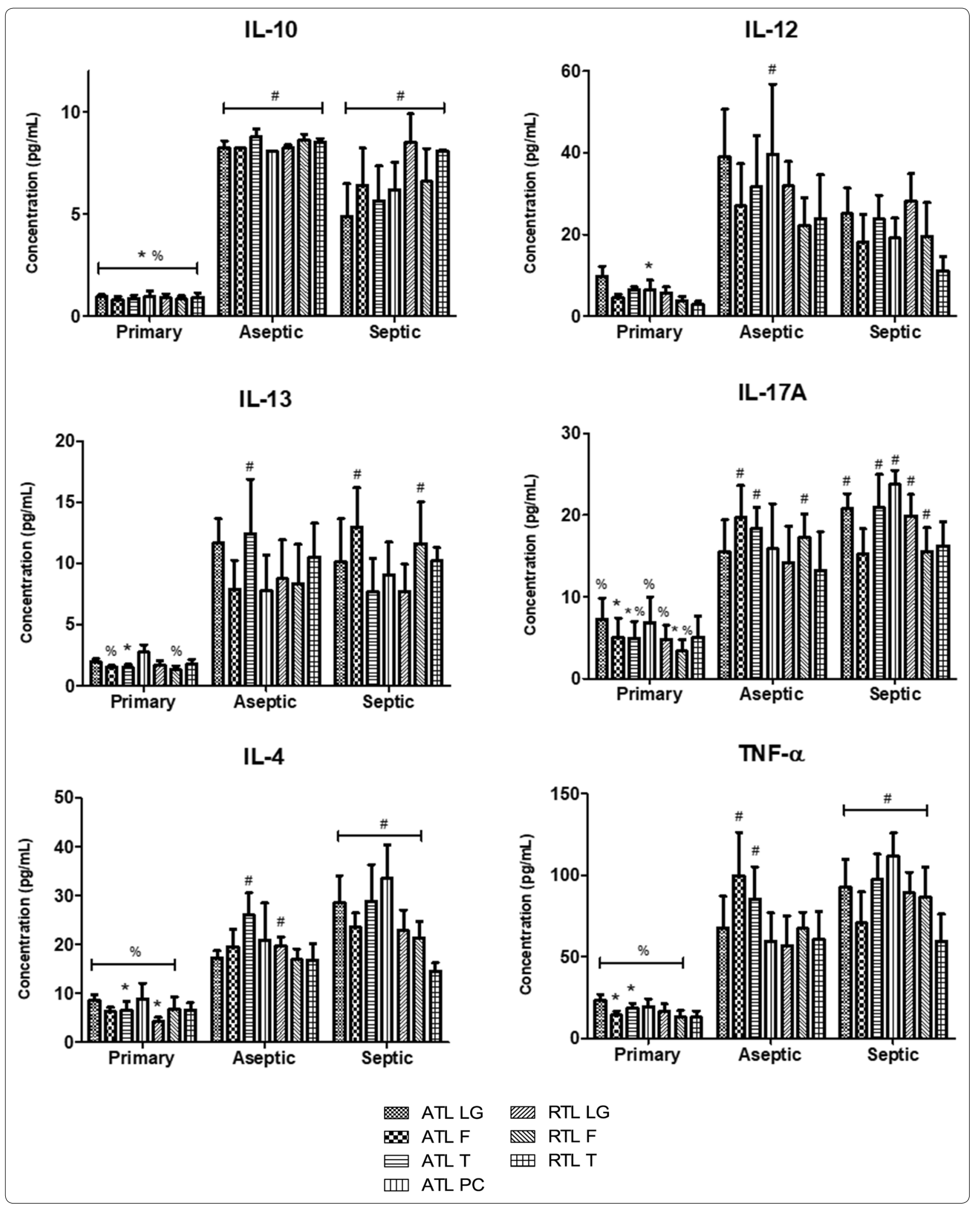




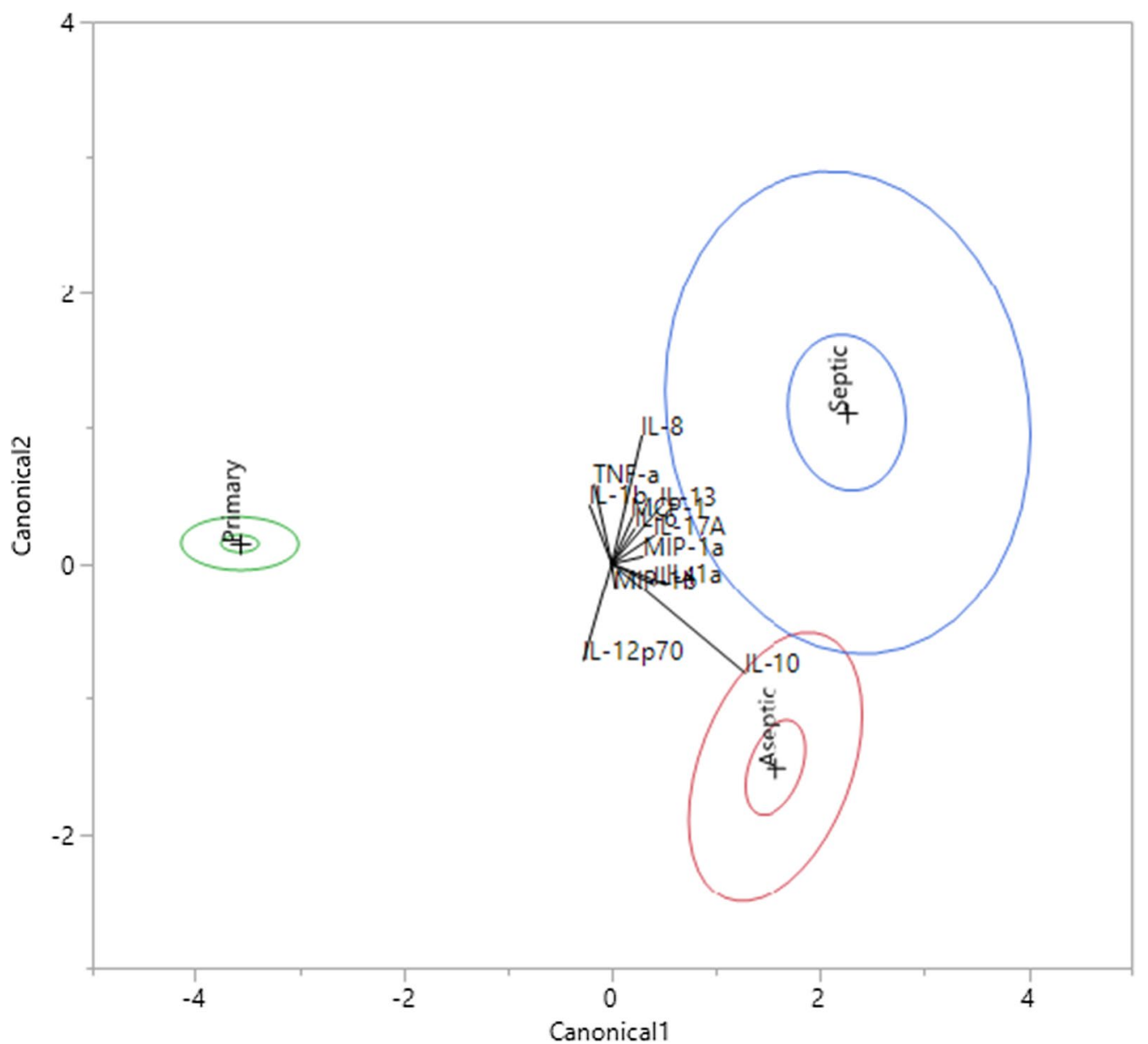

Fig. 4 Quadratic discriminant analysis (QDA) revealed distinct groupings for primary TKA vs. TKR (aseptic or septic). Cytokines with significant infection-dependent or implant-related elevations via two-way ANOVA were analyzed via quadratic discriminant analysis. Canonical scores for each cytokine (covariate) were calculated, and the 95\% confidence interval is shown for primary TKA (green), aseptic TKR (red), and septic TKR (blue). The + symbol represents the mean of each group. Biplot rays describe the degree of association of a certain cytokine with canonical variables

This study focused on defining the tissue-level cytokine environment and modulation in response to implants and infection across several anatomical locations. Many human inflammatory cytokines have been implicated in the systemic response to implants (i.e., in serum/plasma) [43-45] and now aid in diagnosis of infection [46, 47]. However, this investigation is the first, to our knowledge, to assess multiple tissue locations surrounding the joint to address implant-related vs. infection-specific responses. Seven cytokines were identified as infectionspecific, showing elevated concentrations in the septic TKR cohort compared to both the aseptic TKR and primary TKA cohorts: IL-1 $\alpha$, IL-1 $\beta$, IL-6, IL-8, MCP-1, MIP- $1 \alpha$, and MIP- $1 \beta(p<0.05)$. Several of these cytokines have illustrated their utility in the literature for diagnosis of PJI (i.e. IL-1 $\alpha$, IL-1 $\beta$, IL-6, IL-8), but this is the first instance of their investigation on a tissue level [30-33]. Generally, these seven cytokines were elevated in ATL layer tissues compared to RTL layer tissues, which brings to light the importance of proximity to joint in dictating cytokine response. Pro-inflammatory cytokines like IL-1 $\alpha$, IL-1 $\beta$, IL- 6 , and IL- 8 have been noted for their roles in early infection response, producing a warning signal of pathogen invasion, and this response was present in septic TKR tissues [48, 49]. These early cytokine indicators recruit factors like MCP-1, MIP- $1 \alpha$, and MIP- $1 \beta$ that propagate the response to pathogens through Th1 and Th2 immune signaling cascades [50, 51]. Six cytokines were identified as exhibiting a response due to implantation, with elevations in aseptic and septic TKR vs. primary TKA: IL-10, IL-12p70, IL-13, IL-17A, IL-4, and TNF- $\alpha(\mathrm{p}<0.05)$. The elevated concentrations of these cytokines in localized tissues highlighted the degree of inflammation in implanted joints, without the presence of infection, which is likely due to the presence of a foreign body. The implant-related inflammation 
Table 2 Pairwise pearson correlation values between cytokine concentrations and age, sex, and BMI

\begin{tabular}{|c|c|c|c|c|c|c|c|c|c|}
\hline \multirow[t]{2}{*}{ Cytokine } & \multicolumn{3}{|c|}{ Primary TKA } & \multicolumn{3}{|c|}{ Aseptic TKR } & \multicolumn{3}{|c|}{ Septic TKR } \\
\hline & Age & Sex & BMI & Age & Sex & BMI & Age & Sex & BMI \\
\hline IL-1a & 0.00 & 0.47 & 0.28 & 0.20 & 0.22 & -0.54 & -0.15 & 0.25 & -0.05 \\
\hline $\mid L-1 \beta$ & -0.19 & -0.06 & -0.04 & -0.30 & -0.30 & 0.08 & 0.03 & 0.26 & 0.05 \\
\hline IL-6 & 0.00 & -0.09 & -0.12 & 0.03 & -0.19 & -0.21 & 0.00 & 0.22 & -0.02 \\
\hline IL-8 & 0.03 & 0.59 & 0.25 & 0.06 & -0.07 & -0.32 & -0.13 & 0.14 & -0.14 \\
\hline MCP-1 & 0.04 & 0.13 & 0.14 & -0.18 & -0.44 & 0.45 & -0.07 & 0.20 & -0.15 \\
\hline MIP-1a & -0.10 & 0.13 & -0.04 & -0.05 & -0.30 & 0.09 & 0.33 & 0.08 & -0.13 \\
\hline MIP-1 $\beta$ & -0.04 & 0.19 & 0.03 & 0.23 & -0.06 & -0.38 & 0.21 & 0.13 & -0.16 \\
\hline IL-10 & -0.26 & 0.29 & 0.17 & -0.31 & 0.00 & -0.20 & -0.11 & -0.25 & 0.30 \\
\hline IL-12p70 & -0.06 & 0.02 & 0.03 & -0.03 & 0.49 & -0.32 & -0.30 & -0.18 & 0.33 \\
\hline IL-13 & -0.19 & 0.49 & 0.28 & -0.06 & 0.41 & -0.06 & -0.22 & -0.23 & 0.22 \\
\hline IL-17A & 0.00 & -0.08 & -0.31 & 0.31 & 0.85 & -0.45 & -0.34 & 0.06 & 0.32 \\
\hline IL-4 & -0.32 & 0.20 & 0.29 & 0.34 & 0.35 & -0.55 & 0.21 & 0.02 & -0.11 \\
\hline TNF-a & -0.22 & 0.34 & 0.25 & 0.45 & 0.72 & -0.38 & -0.45 & -0.12 & 0.25 \\
\hline
\end{tabular}

The pairwise correlation values are listed for each of the three groups: primary TKA, aseptic TKR, and septic TKR. Pearson correlation values are rounded to two decimal places. No correlations were found to be significant at the $p<0.05$ level after Bonferroni's correction

reflected less of the macrophage activation present in the septic TKR group, but exhibited elevation in antiinflammatory cytokines like IL-10, IL-4, and IL-13 frequently associated with bone healing [7]. IL-17A and IL-12p70 have both pro- and anti-inflammatory roles, but the specific contributions to foreign body response are not well understood. Elevation of these cytokines, as well as TNF- $\alpha$, implies there may be dysregulation of inflammatory response due to implant. These cytokines were not significantly elevated in infection at the $\mathrm{p}<0.05$ level, so they may be considered as indicators of aseptic or chronic inflammation that could be addressed with future research associated with TKA. Further, QDA analysis illustrated that cytokine profiles are distinct between all three cohorts, but there is significant overlap in the 95\% confidence intervals of aseptic TKR and septic TKR. While there are several cytokines that distinctly separate these two cohorts, this analysis indicated that the degree of inflammation experienced between these groups is comparable. This finding agrees with the clinical decision to address inflammation and perform revision surgery, and these markers (IL-10, IL-12p70, IL-13, IL-17A, IL-4, and TNF- $\alpha$ ) may show promise as helpful diagnostic monitoring markers for patients suffering from inflammatory complications in the absence of infection.

While this study had several limitations (i.e., single operating surgeon, heterogeneous cohort of patients, pathogen variability), it represents a novel characterization of tissue-level cytokines across different anatomical locations in response to implants as well as infectionspecific inflammation. Further, these cytokines may give insight into the health of localized tissue following these procedures. Additionally, it highlights the utility of investigating a truly localized view of tissue health, by testing tissues surrounding the joint following these procedures; this approach could aid clinicians' understanding of the localized tissue to better support clinical decision making. At the time of publication, all patients had reached at least the 1-year post-operative follow up without need for revision, with no recurrent infections, and the predictive value of these cytokines for successful surgical outcomes is of interest in future studies. These cytokines could potentially be incorporated to intra-operatively assess the amount of inflammation during surgery, providing information in real time about the viability of tissues for debridement. A more focused investigation of infection-specific markers IL-1 $\alpha$, IL-1 $\beta$, IL-6, IL-8, MCP1, MIP- $1 \alpha$, and MIP-1 $\beta$ could provide insight into the power of these cytokines to discriminate aseptic vs. septic tissues.

\section{Conclusions}

In conclusion, this exploratory pilot study identified several cytokines that exhibited higher concentrations in response to implant-related and infection-specific post-operative inflammation. Some of these cytokines have been previously implicated in chronic inflammation and infection following TKA/TKR on a systemic level [11, 12, 30-33], and this study confirmed this trend on a localized tissue level. Literature has already illustrated that local inflammation is much more important for early post-operative recovery for a few markers [6], and this study expanded on that knowledge to provide an extended view of inflammatory cytokines 
involved in tissue health. Future studies will build off this localized tissue-level information to investigate the mechanisms of dysregulation observed between the groups.

Overall, investigating the localized tissue-level cytokines to understand implant-related and infectionspecific inflammatory complications following knee arthroplasty may offer insight into localized response and provide new diagnostic and therapeutic options. Although this study did not control for age, sex, or BMI, these cytokines were not significantly correlated to these variables, suggesting these were not confounding factors (Table 2) in this study. Future work will focus on studies to include a larger cohort of patients to control for a variety of factors, including age, sex, BMI, and comorbidities. Ultimately, this study provided a basis to study these cytokines in surgical scenarios as a quantitative means to verify clinical decisions. More research is needed to confirm potential localized biomarkers that may be associated with chronic inflammation. In the future, larger cohort studies could utilize the infection-specific biomarkers for retrospective review of patient outcomes throughout the rehabilitation process.

\begin{abstract}
Abbreviations
TKA: Total knee arthroplasty; TKR: Total knee revision; PJl: Prosthetic joint infection; IL: Interleukin; TNF-a: Tumor necrosis factor-alpha; IRB: Institutional Review Board; BMI: Body mass index; MSIS: Musculoskeletal Infection Society; MSSA: Methicillin-sensitive Staphylococcus aureus; CRP: C-reactive protein; ATL: Adjacent tissue layer; RTL: Radial tissue layer; F: Medial femoral condyle; T: Medial tibial plateau; LG: Lateral gutter; PC: Posterior capsule; PBS: Phosphatebuffered saline; 4-PL: Four-parameter logistic; 5-PL: Five-parameter logistic; LLOQ: Lower limit of quantitation; ULOQ: Upper limit of quantitation; IQR: Interquartile range; ANOVA: Analysis of variance; SEM: Standard error of the mean; MCP-1: Monocyte chemoattractant protein-1; MIP-1 a: Macrophage inflammatory protein-1 alpha; MIP-1 $\beta$ : Macrophage inflammatory protein-1 beta; GM-CSF: Granulocyte-macrophage colony-stimulating factor; IFN-a: Interferon-alpha; IFN-ץ: Interferon-gamma; IP-10: Interferon gamma-induced protein 10; QDA: Quadratic discriminant analysis.
\end{abstract}

\section{Acknowledgements}

The authors would like to thank Jennifer Eicher for her work in recruitment of patients for the study. Further, we would like to thank Suzanne Danley for her editorial suggestions. We would like to thank Clara Warner for developing graphic design images of the knee anatomy.

\section{Authors' contributions}

The following authors have made substantial contributions to conception and design of the study: NP, MD, JB. The following authors have made substantial contributions to acquisition of data or analysis and interpretation of data: NP, $M D$, JB. The following authors have been involved in drafting the manuscript or revising it critically for important intellectual content: NP, JP, MD, JB. The following authors have given final approval for the version to be published and agree to be accountable for all aspects of the work in ensuring that questions related to the accuracy or integrity of any part of the work are appropriately investigated and resolved: NP, JP, MD, JB. All authors read and approved the final manuscript.

\section{Funding}

Funding for this work was provided by the National Institutes of Health [K08AR073921], and institutional support provided by the West Virginia University School of Medicine, Department of Orthopaedics.

\section{Availability of data and materials}

The datasets generated and/or analyzed during the current study are not publicly available to protect the privacy of the participants but are available from the corresponding author on reasonable request.

\section{Ethics approval and consent to participate}

Following Institutional Review Board (IRB) approval (IRB Protocol $\# 1709745853$ ) and patient consent, six patients undergoing primary total knee arthroplasty (TKA) and eleven patients undergoing total knee revision (TKR) procedures participated in the study. Ethics approval was granted by West Virginia University Institutional Review Board. Subjects were recruited over a 12-month period.

\section{Consent for publication}

Not applicable.

\section{Competing interests}

The authors declare that they have no competing interests.

\section{Author details}

${ }^{1}$ C. Eugene Bennett Department of Chemistry, West Virginia University, 64 Medical Center Drive, 3900 HSS, P.O. Box 9196, Morgantown, WV 26506-9196, USA. ${ }^{2}$ Department of Orthopaedics, West Virginia University School of Medicine, Morgantown, WV, USA. ${ }^{3}$ Department of Physiology and Pharmacology, West Virginia University, Morgantown, WV, USA.

Received: 9 January 2020 Accepted: 27 August 2020

Published online: 31 August 2020

\section{References}

1. Schulte W, Bernhagen J, Bucala R. Cytokines in sepsis: potent immunoregulators and potential therapeutic targets_-an updated view. Mediat Inflamm. 2013:2013:165974. https://doi.org/10.1155/2013/165974.

2. Dinarello CA. Historical insights into cytokines. Eur J Immunol. 2007;37(Suppl 1):S34-45. https://doi.org/10.1002/eji.200737772.

3. Turner MD, Nedjai B, Hurst T, Pennington DJ. Cytokines and chemokines: at the crossroads of cell signalling and inflammatory disease. Biochim Biophys Acta. 2014;1843(11):2563-82. https://doi.org/10.1016/j.bbamc r.2014.05.014

4. Miller RE, Miller RJ, Malfait AM. Osteoarthritis joint pain: the cytokine connection. Cytokine. 2014;70(2):185-93. https://doi.org/10.1016/j. cyto.2014.06.019.

5. Lisowska B, Maśliński W, Małdyk P, Zabek J, Baranowska E. The role of cytokines in inflammatory response after total knee arthroplasty in patients with rheumatoid arthritis. Rheumatol Int. 2008;28(7):667-71. https://doi.org/10.1007/s00296-007-0508-1.

6. Ugraş AA, Kural C, Kural A, Demirez F, Koldaş M, Cetinus E. Which is more important after total knee arthroplasty: local inflammatory response or systemic inflammatory response? Knee. 2011;18(2):113-6. https://doi. org/10.1016/j.knee.2010.03.004.

7. Rodriguez A, Meyerson H, Anderson JM. Quantitative in vivo cytokine analysis at synthetic biomaterial implant sites. J Biomed Mater Res A. 2009;89(1):152-9. https://doi.org/10.1002/jbm.a.31939.

8. Si HB, Yang TM, Zeng Y, et al. Correlations between inflammatory cytokines, muscle damage markers and acute postoperative pain following primary total knee arthroplasty. BMC Musculoskelet Disord. 2017;18(1):265. https://doi.org/10.1186/s12891-017-1597-y.

9. Shah K, Mohammed A, Patil S, McFadyen A, Meek RM. Circulating cytokines after hip and knee arthroplasty: a preliminary study. Clin Orthop Relat Res. 2009;467(4):946-51. https://doi.org/10.1007/s1199 9-008-0562-3.

10. Ackerman IN, Bohensky MA, Zomer E, et al. The projected burden of primary total knee and hip replacement for osteoarthritis in Australia to the year 2030. BMC Musculoskelet Disord. 2019;20(1):90. https://doi. org/10.1186/s12891-019-2411-9.

11. Wylde V, Beswick A, Bruce J, Blom A, Howells N, Gooberman-Hill R. Chronic pain after total knee arthroplasty. EFORT Open Rev. 2018;3(8):461-70. https://doi.org/10.1302/2058-5241.3.180004. 
12. Lim HA, Song EK, Seon JK, Park KS, Shin YJ, Yang HY. Causes of aseptic persistent pain after total knee arthroplasty. Clin Orthop Surg. 2017;9(1):50-6. https://doi.org/10.4055/cios.2017.9.1.50.

13. Parvizi J, Gehrke T, Infection ICGoPJ. Definition of periprosthetic joint infection. J Arthroplasty. 2014;29(7):1331. https://doi.org/10.1016/j. arth.2014.03.009.

14. Tande AJ, Patel R. Prosthetic joint infection. Clin Microbiol Rev. 2014;27(2):302-45. https://doi.org/10.1128/CMR.00111-13.

15. Li C, Renz N, Trampuz A. Management of periprosthetic joint infection. Hip Pelvis. 2018;30(3):138-46. https://doi.org/10.5371/hp.2018.30.3.138.

16. Currie HN, Loos MS, Vrana JA, Dragan K, Boyd JW. Spatial cytokine distribution following traumatic injury. Cytokine. 2014;66(2):112-8. https://doi. org/10.1016/j.cyto.2014.01.001.

17. Han AA, Currie HN, Loos MS, et al. Spatiotemporal phosphoprotein distribution and associated cytokine response of a traumatic injury. Cytokine. 2016;79:12-22. https://doi.org/10.1016/j.cyto.2015.12.006.

18. Hauser CJ, Zhou X, Joshi P, et al. The immune microenvironment of human fracture/soft-tissue hematomas and its relationship to systemic immunity. J Trauma. 1997;42(5):895-903. https://doi.org/10.1097/00005 373-199705000-00021 (discussion 903-4)

19. Hayden FG, Fritz R, Lobo MC, Alvord W, Strober W, Straus SE. Local and systemic cytokine responses during experimental human influenza $\mathrm{A}$ virus infection. Relation to symptom formation and host defense. J Clin Invest. 1998;101(3):643-9. https://doi.org/10.1172/JCl1355.

20. Cuingnet R, Rosso C, Chupin M, et al. Spatial regularization of SVM for the detection of diffusion alterations associated with stroke outcome. Med Image Anal. 2011;15(5):729-37. https://doi.org/10.1016/j.media 2011.05.007.

21. Clough GF, Jackson CL, Lee JJ, Jamal SC, Church MK. What can microdialysis tell us about the temporal and spatial generation of cytokines in allergen-induced responses in human skin in vivo? J Invest Dermatol. 2007;127(12):2799-806. https://doi.org/10.1038/sj.jid.5700930.

22. Postler A, Lützner C, Beyer F, Tille E, Lützner J. Analysis of total knee arthroplasty revision causes. BMC Musculoskelet Disord. 2018;19(1):55. https:// doi.org/10.1186/s12891-018-1977-y.

23. Imamura M, Ezquerro F, Marcon Alfieri F, et al. Serum levels of proinflammatory cytokines in painful knee osteoarthritis and sensitization. Int J Inflamm. 2015;2015:329792. https://doi.org/10.1155/2015/329792.

24. Usher KM, Zhu S, Mavropalias G, Carrino JA, Zhao J, Xu J. Pathological mechanisms and therapeutic outlooks for arthrofibrosis. Bone Res. 2019;7:9. https://doi.org/10.1038/s41413-019-0047-x.

25. Arango Duque G, Descoteaux A. Macrophage cytokines: involvement in immunity and infectious diseases. Front Immunol. 2014;5:491. https://doi. org/10.3389/fimmu.2014.00491.

26. Chaudhry H, Zhou J, Zhong Y, et al. Role of cytokines as a double-edged sword in sepsis. In Vivo. 2013;27(6):669-84.

27. Cavaillon JM, Adib-Conquy M, Fitting C, Adrie C, Payen D. Cytokine cascade in sepsis. Scand J Infect Dis. 2003;35(9):535-44. https://doi. org/10.1080/00365540310015935.

28. Ma L, Zhang $\mathrm{H}$, Yin $\mathrm{YL}$, et al. Role of interleukin- 6 to differentiate sepsis from non-infectious systemic inflammatory response syndrome. Cytokine. 2016;88:126-35. https://doi.org/10.1016/j.cyto.2016.08.033.

29. Shahzad A, Knapp M, Lang I, Köhler G. Interleukin 8 (IL-8)—a universal biomarker? Int Arch Med. 2010;3:1 1. https://doi. org/10.1186/1755-7682-3-11.

30. Delanois RE, Mistry JB, Gwam CU, Mohamed NS, Choksi US, Mont MA. Current epidemiology of revision total knee arthroplasty in the United States. J Arthroplasty. 2017;32(9):2663-8. https://doi.org/10.1016/j. arth.2017.03.066.

31. Saleh A, George J, Faour M, Klika AK, Higuera CA. Serum biomarkers in periprosthetic joint infections. Bone Joint Res. 2018;7(1):85-93. https:// doi.org/10.1302/2046-3758.71.BJR-2017-0323.

32. Frangiamore SJ, Siqueira MB, Saleh A, Daly T, Higuera CA, Barsoum WK. Synovial cytokines and the MSIS criteria are not useful for determining infection resolution after periprosthetic joint infection explantation. Clin Orthop Relat Res. 2016;474(7):1630-9. https://doi.org/10.1007/s1199 9-016-4710-x.
33. Rider P, Carmi Y, Cohen I. Biologics for targeting inflammatory cytokines, clinical uses, and limitations. Int J Cell Biol. 2016;2016:9259646. https:// doi.org/10.1155/2016/9259646.

34. Hulse RE, Kunkler PE, Fedynyshyn JP, Kraig RP. Optimization of multiplexed bead-based cytokine immunoassays for rat serum and brain tissue. J Neurosci Methods. 2004;136(1):87-988. https://doi.org/10.1016/j.jneum eth.2003.12.023.

35. Schäfer J, Strimmer K. A shrinkage approach to large-scale covariance matrix estimation and implications for functional genomics. Stat Appl Genet Mol Biol. 2005;4:32. https://doi.org/10.2202/1544-6115.1175.

36. Bland JM, Altman DG. Multiple significance tests: the Bonferroni method. BMJ. 1995;310(6973):170. https://doi.org/10.1136/bmj.310.6973.170.

37. Obanda V, Omondi GP, Chiyo PI. The influence of body mass index, age and sex on inflammatory disease risk in semi-captive Chimpanzees. PLoS ONE. 2014;9(8):e104602. https://doi.org/10.1371/journal.pone.0104602.

38. Frasca D, Blomberg BB, Paganelli R. Aging, obesity, and inflammatory agerelated diseases. Front Immunol. 2017;8:1745. https://doi.org/10.3389/ fimmu.2017.01745.

39. Vendrell J, Broch M, Vilarrasa N, et al. Resistin, adiponectin, ghrelin, leptin, and proinflammatory cytokines: relationships in obesity. Obes Res. 2004;12(6):962-71. https://doi.org/10.1038/oby.2004.118.

40. Lee YS, Koo KH, Kim HJ, et al. Synovial fluid biomarkers for the diagnosis of periprosthetic joint infection: a systematic review and meta-analysis. J Bone Joint Surg Am. 2017;99(24):2077-84. https://doi.org/10.2106/ JBJS.17.00123.

41. Shahi A, Parvizi J. The role of biomarkers in the diagnosis of periprosthetic joint infection. EFORT Open Rev. 2016;1 (7):275-8. https://doi. org/10.1302/2058-5241.1.160019.

42. Chen MF, Chang CH, Yang LY, et al. Synovial fluid interleukin-16, interleukin-18, and CRELD2 as novel biomarkers of prosthetic joint infections. Bone Joint Res. 2019;8(4):179-88. https://doi.org/10.1302/2046-3758.84. BJR-2018-0291.R1.

43. Fassbender K, Dempfle CE, Mielke O, et al. Proinflammatory cytokines: indicators of infection in high-risk patients. J Lab Clin Med. 1997;130(5):535-9. https://doi.org/10.1016/s0022-2143(97)90131-1.

44. Matsumoto $\mathrm{H}$, Ogura $\mathrm{H}$, Shimizu K, et al. The clinical importance of a cytokine network in the acute phase of sepsis. Sci Rep. 2018;8(1):13995. https://doi.org/10.1038/s41598-018-32275-8.

45. Ramnath RD, Weing $\mathrm{S}$, He M, et al. Inflammatory mediators in sepsis: cytokines, chemokines, adhesion molecules and gases. J Organ Dysfunct. 2009;2(2):80-92. https://doi.org/10.1080/17471060500435662.

46. Ye Q, Shao WX, Xu XJ, Yang YZ. The clinical application value of cytokines in treating infectious diseases. PLoS ONE. 2014;9(6):e98745. https://doi. org/10.1371/journal.pone.0098745.

47. Monastero RN, Pentyala S. Cytokines as biomarkers and their respective clinical cutoff levels. Int J Inflamm. 2017;2017:4309485. https://doi. org/10.1155/2017/4309485.

48. Tanaka T, Narazaki M, Kishimoto T. IL-6 in inflammation, immunity, and disease. Cold Spring Harb Perspect Biol. 2014;6(10):a016295. https://doi. org/10.1101/cshperspect.a016295.

49. Bergsbaken T, Fink SL, Cookson BT. Pyroptosis: host cell death and inflammation. Nat Rev Microbiol. 2009;7(2):99-109. https://doi.org/10.1038/ nrmicro2070.

50. Trumpfheller C, Tenner-Racz K, Racz P, Fleischer B, Frosch S. Expression of macrophage inflammatory protein (MIP)-1alpha, MIP-1 beta, and RANTES genes in lymph nodes from HIV+ individuals: correlation with a Th1-type cytokine response. Clin Exp Immunol. 1998;112(1):92-9. https://doi.org/1 0.1046/j.1365-2249.1998.00555.x.

51. Ruiz Silva M, van der Ende-Metselaar $\mathrm{H}$, Mulder HL, Smit JM, RodenhuisZybert IA. Mechanism and role of MCP-1 upregulation upon chikungunya virus infection in human peripheral blood mononuclear cells. Sci Rep. 2016;6:32288. https://doi.org/10.1038/srep32288.

\section{Publisher's Note}

Springer Nature remains neutral with regard to jurisdictional claims in published maps and institutional affiliations. 